\title{
LIGHT FIDELITY FOR POSITION DETECTION
}

\author{
Sauvik Bal ${ }^{1}$ \\ ${ }^{I}$ Department of Computer Science \& Engineering, Institute of Engineering \& Management, West Bengal, India
}

\begin{abstract}
Li Fi (Light Fidelity) is a wireless communication system by which we can access the internet. Li Fi uses light for communication. So it is very easy to use in practical life. Here I propose a technique by using which we can monitoring any li-fi enable component. Also by using this technique we can create a very clear view of road map. We can also use this method for security purpose. . Suppose a person/car walking through the road. By using this method we can identify where the person is. The road side lamp posts are used as a source of light. Every person should have a photo detector device. That is the receiver. So easily connection will be made between road side lamp and that device. When a moving person is crossed a particular area and entered a new area, the place notification is automatically updated in his device.
\end{abstract}

Keywords: Wi Fi, Li Fi, VLC, LED.

\section{INTRODUCTION}

$\mathrm{Li} \mathrm{Fi} \mathrm{is} \mathrm{a} \mathrm{communication} \mathrm{system} \mathrm{where} \mathrm{light} \mathrm{is} \mathrm{used} \mathrm{as} \mathrm{a}$ carrier. This Li Fi technique is similar as Wi Fi but here instead of radio waves, light is used as a carrier. VLC (Visible Light Communication) system is used in this technique [2]. Li fi technology support high speed data transmission wirelessly. Also in Li Fi technology there is no use of wire, so beside the road it is very easy to use for the users those who are passing through the road. Here we discuss the method by which we monitoring the movement of a person/car on the road of a city. In this technology the intensity of light which is used is much faster than the human eye can follow [2].

\section{LI FI}

Now a days internet users are increasing rapidly. Every person try to use Wi Fi technology, so the radio spectrum is congested. Li Fi technology is very much necessary for future. In $\mathrm{Li} \mathrm{Fi}$ technology, information strings are generated by using 0 and 1 . This mechanism is very fast so that human eye cannot notice ON \& OFF. When light is ON, it generates 1 and when it is OFF, it generates 0 . In $\mathrm{Li} \mathrm{Fi}$ method visible spectrum is used.

\section{LI FI FOR IDENTIFY}

Here we use this technology to identify a person/ car which is passing through the road. In this scenario all the lamp post are working as a source and the lights of the lamp post are LED. Each and every lamp post has a particular area which is covered by that light. Each lamp post has a unique name. There would be photo detector device for receiving purpose. When the light is reflected on it, the signal are processed and information will be shown to the receiver. Every lamp post has a unique name. The information about the lamp post also would be shown to the receiver. If anybody want to monitoring then it would be very easy to him. Here I have discuss the method in step by step.

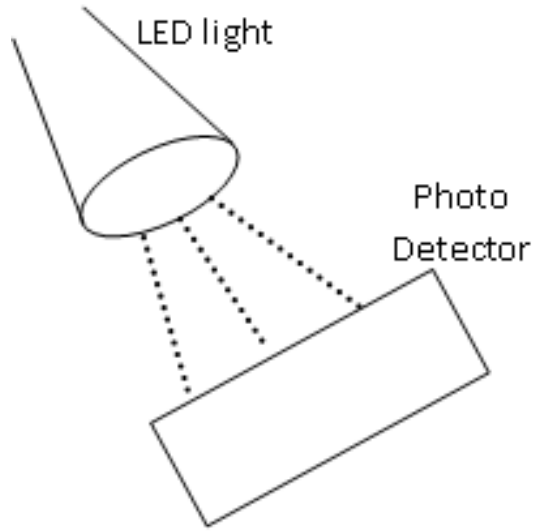

Fig -1: LED (Source) \& Photo Detector (Receiver)

\subsection{Proposed Method}

- All the light post on road side should be Li-fi enabled. That is every lights (LED) of the light posts are working as a source [3].

- A photodiode would be used as a receiver. This device should be present in every people those who are passing through the road.

- By using that device every person are able to connect with the lamp post (source).

- $\quad$ Every Li Fi enabled lamp post should have a unique name.

- So this is easy to identify which person is in which position.

- $\quad$ Each lamp post covered a particular area.

- When the person crossed a particular lamp post and entered to the area of another post, the area notification is updated on the device.

- If anybody try to monitoring a person/car which is on the road, easily identify them by using this method. 


\subsection{Explanation with Diagram}

Here in Fig. 2, two lamp posts are present named A, B. each lamp post covered a particular area.

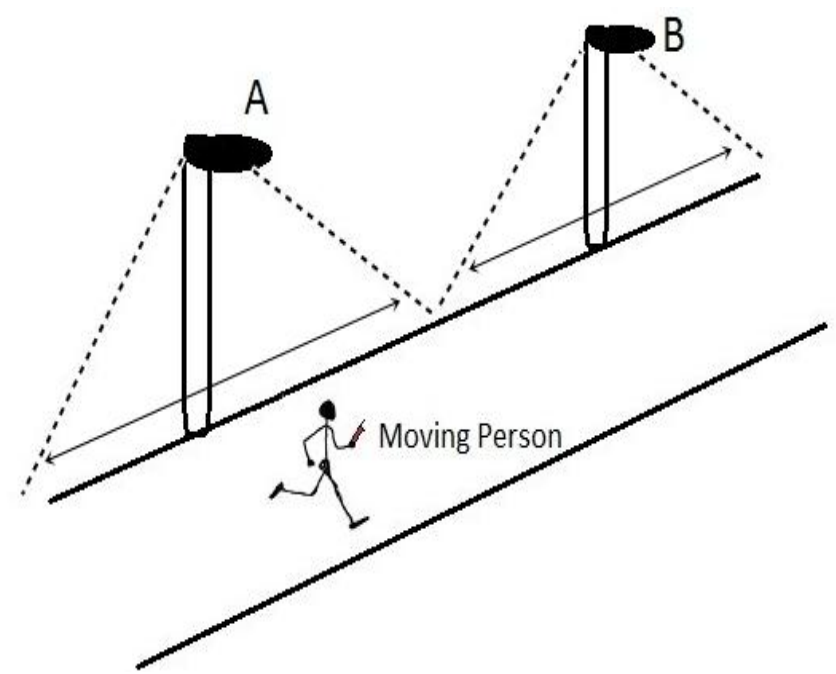

Fig -2: Covered area \& Moving person

Here is a moving person who run over the road. When he is in the area of $\mathrm{A}$, the location is updated in his photodiode device. Every lamp post has a unique name and it is based on the location name. When he cross over the area of $\mathrm{A}$, the connection is automatically disconnected. Because each lamp post has a particular range. When the moving person cross that range, the light will not been reflected on that photodiode.

After crossing the A's range, the person would be on the range of another lamp post, suppose B. on that time the light will be fallen on the photodiode. Then in the similar fashion connection will be established between $\mathrm{B}$ and the receiver. Also the location is updated in the device.

\section{FOR MONITORING}

By using Li Fi technology we can monitoring any movable object. All the road side lamp posts are the source. The lamp posts are interconnected and all are connected to the internet [1]. Now suppose there is a monitor where we can notice the positions of the lamp posts for a particular road. All the lamp posts are considered as a node which has a unique name. By using this concept, we can generate the road map in every city, village etc. If any moving person/car passing through the road, then in the monitoring map, there would be movable node noticed.

In Fig -3, there are some areas represented as A, B, C, D, E, and $\mathrm{F}$. The road side lamp posts are named as per the area name. Suppose there are 4 road side lamp post in the area A. then the unique name of that lamp posts are A1, A2, A3, and A4 which are represented as a node. Similarly the lamp post which are in area B are named as B1, B2, and B3. Similarly there are lamp post present in the different area and all have different unique name.
Primarily there are not any road shown in the monitor. Firstly all the lamp post (node) and their names are shown to the monitor. If join the nodes (lamp posts) by virtual line, we get the road map. After that we create the proper road map. Here some movable objects are shown on the road. In the monitor that also would be represented as a node. In the Fig $-3 \mathrm{~W}, \mathrm{X}, \mathrm{Y}, \mathrm{Z}$ are the node.

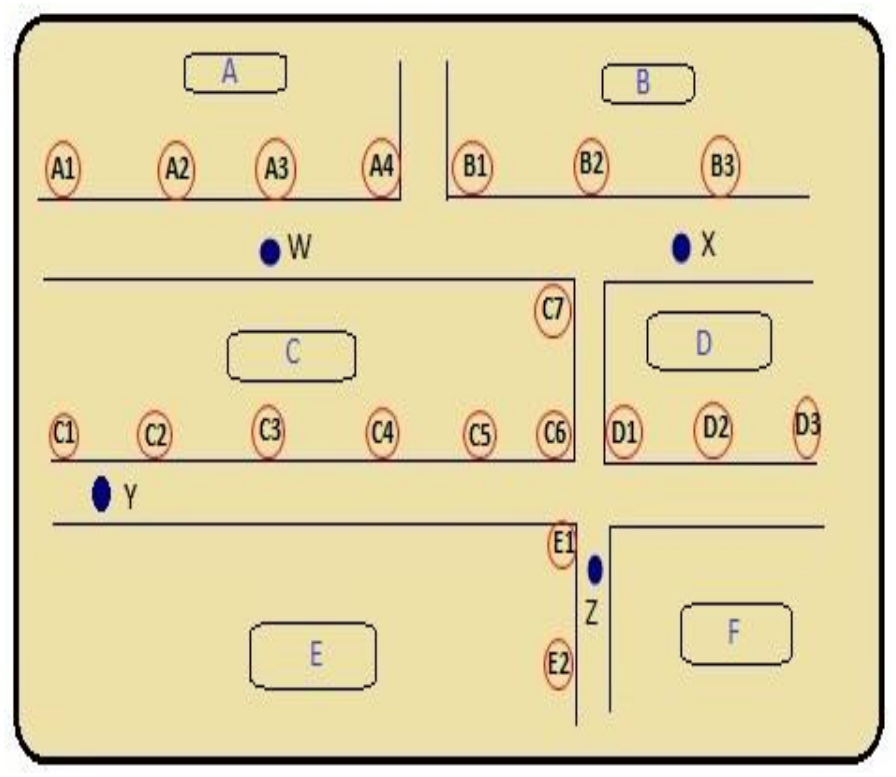

Fig -3: Monitoring Screen

In the Fig -3 node $\mathrm{W}$ (movable object) is nearby A3 node (lamp post). So there is a connection made between A3 and $\mathrm{W}$. On the screen of photo diode of $\mathrm{W}$, the node name/ lamp post name i.e. A3 is shown. When it crossed A3 and entered into the area of $\mathrm{A} 4$, then details of $\mathrm{A} 4$ will be shown. Similar thing is happen for the node $\mathrm{X}, \mathrm{Y}, \mathrm{Z}$.

On the monitoring screen the view is very clear to the user because in this method lamp post working as a source.

\section{ARCHITECTURE}

Now I discuss the architecture of the $\mathrm{Li} F i$ as a position detecting tool.

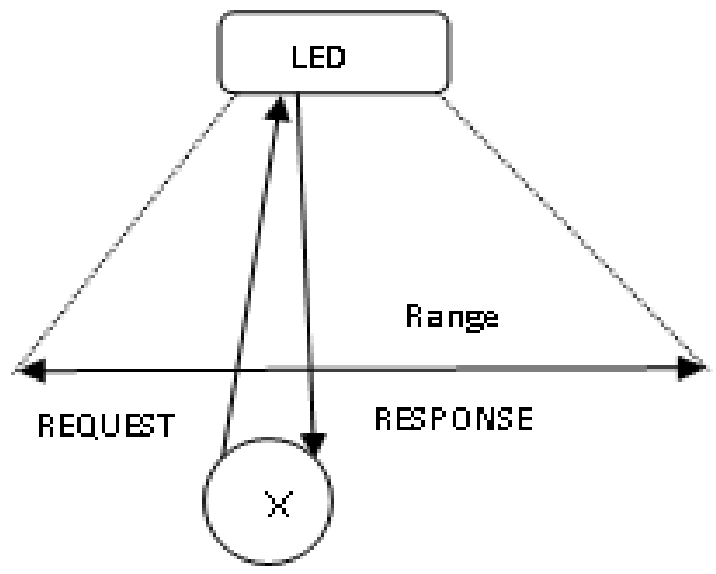

Fig -4: Req. \& Response 
LED is the source attached in the lamp post. Suppose A is the movable object. When it is entered in the area of a particular lamp post, it sends REQUEST to that LED for connection. If RESPONSE come from that source, then connection established. This request-response activity only done when the object is in the range of a particular lamp post area. When the object crossed the range of a lamp post, then the connection with that post will automatically lost. This object will entered in the another area of LED post and in the similar fashion the connection will made.

Now I discuss here what is happen when multiple LED (Source) are present.

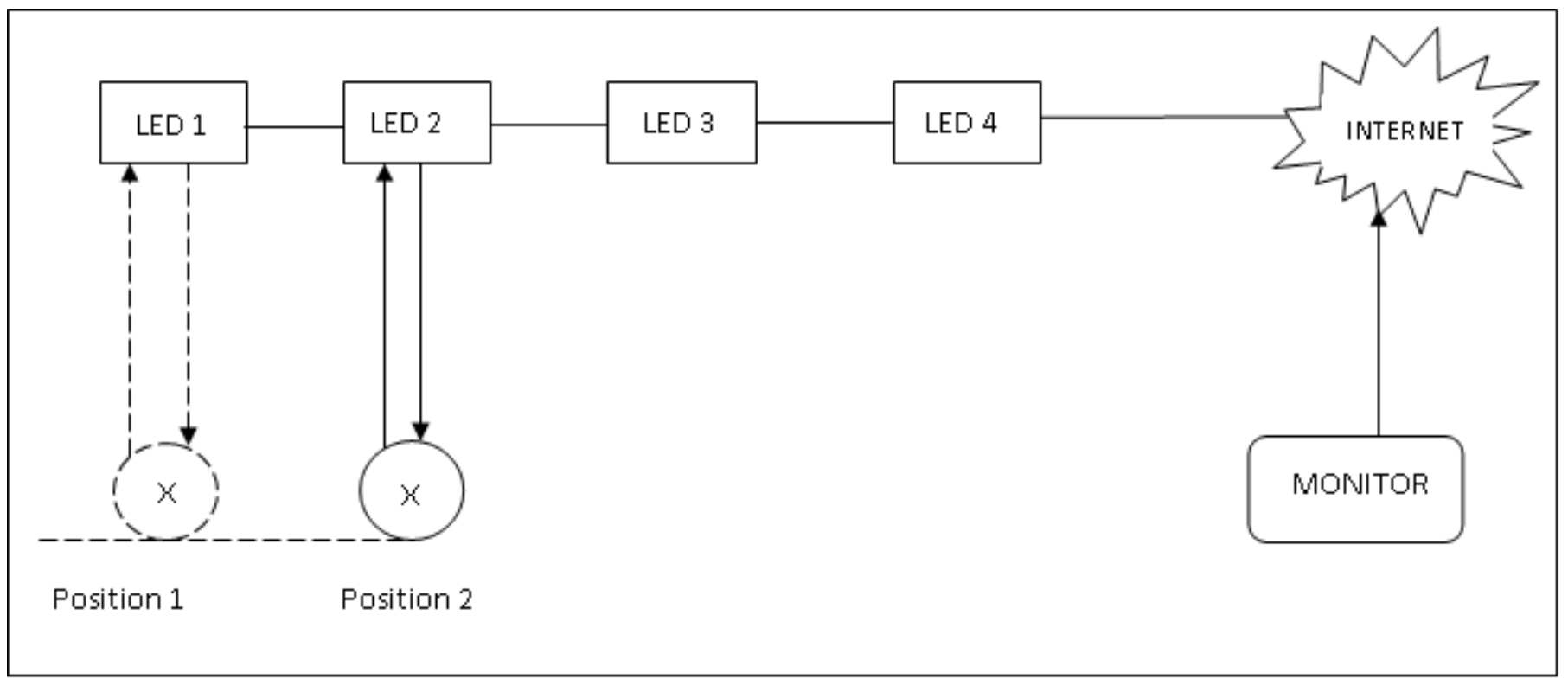

Fig -5: Connection between Sources with Internet with Monitor

Suppose there is a movable object named $\mathrm{X}$ which is crossing over the road.

1. At first $\mathrm{X}$ is in the Position 1 which is in the range of LED 1.

2. It sends request to Led 1 .

3. If network is okay then response come to $\mathrm{X}$ from LED 1.

4. Connection established and data packets are coming from source (LED 1).

5. Details of LED 1 is updated on the screen of $X$.

6. It is assumed that $\mathrm{X}$ is a movable object. So after sometime it crosses the range of LED 1.

7. On that time data packet receiving from LED 1 is stopped and connection lost.

8. The object come in the range of LED 2, here position 2.

9. In the similar fashion it sends request to LED 2 .

10. If all are okay then connection made.

11. The LEDs are interconnected and finally it connect to the internet.

12. Monitor screen is also connected to the internet.

13. If we monitor $\mathrm{x}$, then we have to send request to the internet server.
14. If $\mathrm{X}$ gave the permission then only connection will made and the position of the $X$ will show on the monitor screen.

\section{ADVANTAGES}

In this architecture light is used as a carrier of the data. The source will be ON and OFF to generate the data string. This process is very fast so that human eye cannot differentiate this ON, OFF. Visible light spectrum is not harmful for human body.

We use lamp post as a source. So the people those who are passing through the road can easily access the internet.

We can generate the road map very clearly and very specific manner by using these.

Also we can monitor any movable object by using this technique.

$\mathrm{Li} \mathrm{Fi}$ is cheaper as well as faster than Wi Fi [2]. 


\section{CONCLUSION}

This process would help the people to connect with high speed internet at the time of travelling There is a drawback in Li Fi that light cannot penetrate the brick walls. But if we use Li Fi method in road side lamp post for the people those who are passing through the road, this problem does not hamper the connection process. Another thing is that at a time how many user can be connected with a particular source should not yet identify.

\section{ACKNOWLEDGEMENTS}

I would like to acknowledge all the faculties of IEM, Kolkata those who always extend their hands in all kind of problems regarding this paper. I would also like to thank my family and friends who supported me to prepare this paper.

\section{REFERENCES}

[1]. Rahul R. Sharma1, Raunak, Akshay Sanganal, "Li-Fi Technology Transmission of data through light", IJCTA, Rahul R Sharma et al, Int.J.Computer Technology \& Applications, Vol 5 (1),150-154

[2]. Megha Goyal, Dimple Saproo, Asha Bhagashra, New Epoch of Wireless Communication: Light Fidelity", International Journal of Innovative Research in Computer and Communication Engineering, Vol. 1, Issue 2, April 2013

[3]. Dr. Y.P.Singh, "Critical Technical Aspect and Extensive Research Study of the Light Fidelity - (a Future Communication)", International Journal of IT, Engineering and Applied Sciences Research (IJIEASR) ISSN: 23194413, Volume 2, No. 9, September 2013

[4]. http://visiblelightcomm.com/

\section{BIOGRAPHIE}

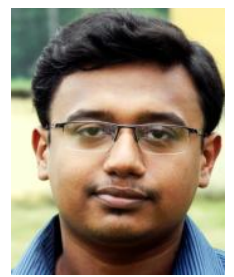

Sauvik Bal, pursuing M.Tech in Computer Science and Engineering and also working as a Teaching Assistant in Institute of Engineering \& Management, Kolkata. E MAIL ID: sauvikbal@gmail.com 